\title{
MACHINE LEARNING APPLICATION FOR OPTIMIZING ASYMMETRICAL REDUCTION OF ACETOPHENONE EMPLOYING COMPLETE CELL OF LACTOBACILLUS SENMAIZUKE AS AN ENVIRONMENTALLY FRIENDLY APPROACH
}

\author{
Osman Taylan $^{a^{*}}$, Mustafa Tahsin Yilmaz ${ }^{\mathrm{a}}$, Mohammed Balubaid ${ }^{\mathrm{a}}$, Rami Alamoudi ${ }^{\mathrm{a}}$,Tahra El-Obeid ${ }^{\mathrm{b}}$, \\ Enes Dertlic, Engin Şahinc, Ahmed Bakhsha ${ }^{a}$, Enrique Herrera-Viedmad \\ $a^{*}$ King Abdulaziz University, Faculty of Engineering, Department of Industrial Engineering, \\ 21589, Jeddah, Saudi Arabia; \\ ${ }^{b}$ Qatar University, QU Health, College of Health Sciences, Human Nutrition Department, 2713, Doha, Qatar; \\ ${ }^{c}$ Bayburt University, Faculty of Engineering, Department of Food Engineering, 69000, Bayburt, Turkey; \\ ${ }^{d}$ University of Granada, Department of Computer Science and Artificial Intelligence, E-18071, Granada, Spain;
}

*Corresponding author Osman Taylan e-mail: otaylan@kau.edu.sa;

Received December 2019; Accepted January 2020; Published February 2020;

DOI: https://doi.org/10.31407/ijees10.117

\begin{abstract}
Recently, optimization of the bioreduction reactions by optimization methodologies has gained special interest as these reactions are affected by several extrinsic factors that should be optimized for higher yields. An important example for these kinds of reactions is the complete cell implications for the bioreduction of prochiral ketones in which the culture parameters play crucial roles. Such biocatalysts provide environmentally friendly and clean methodology to perform reactions under mild conditions with high conversion rates. In the present work, at the first step the Lactobacillus senmaizuke was isolated from sourdough and the complete cell application of Lactobacillus senmaizuke for the bioreduction of acetophenone was optimized by an Artificial Neural networks (ANNs) to achieve the highest enantiomeric excess $(E E, \%)$. The culture parameters, $\mathrm{pH}$, temperature, incubation period and agitation speed were the experimental factors that were optimized to maximize $E E(\%)$ by machine learning algorithm of Artificial Intelligence modeling and the best conditions to maximize EE (95.5\%) were calculated to be $\mathrm{pH}$ of 5.7, temperature of $35^{\circ} \mathrm{C}$, incubation period of $76 \mathrm{~h}$ and agitation speed of $240 \mathrm{rpm}$ with very low sum of squared error value $(0.611236 \%)$ to bioreduce acetophenone using complete cell of Lactobacillus senmaizuke as a sourdough isolate GRAS microbial species. Accordingly, The ANN was employed to correctly establish the enantiomeric excess values of the specimen with an average absolute error $0.080739 \%$.
\end{abstract}

Keywords: Sourdough, Asymmetric bioreduction, Biocatalyst, Chirality, Machine learning, ANNs, Biotransformation 